\title{
On Representations of $\operatorname{sl}(n, \mathbb{C})$ Compatible with a $\mathbb{Z}_{2}$-grading
}

\author{
M. Havlíček, E. Pelantová, J. Tolar
}

\begin{abstract}
This paper extends existing Lie algebra representation theory related to Lie algebra gradings. The notion of a representation compatible with a given grading is applied to finite-dimensional representations of $\operatorname{sl}(n, \mathbb{C})$ in relation to its $\mathbb{Z}_{2}$-gradings. For representation theory of $\operatorname{sl}(n, \mathbb{C})$ the Gel'fand-Tseitlin method turned out very efficient. We show that it is not generally true that every irreducible representation can be compatibly graded.
\end{abstract}

\section{Introduction}

Contractions of Lie algebras, of interest in connecting physical theories, are traditionally understood as limit procedures through which Lie algebras are modified into different, non-isomorphic Lie algebras [5, 8]. Nevertheless, for many physical applications, especially in quantum theory, representations of Lie algebras are important. It should be noted that contractions usually produce non-compact Lie algebras whose unitary representations are infinite-dimensional.

Remaining inside the framework of Lie algebras, a completely different notion of graded contractions was proposed in [11]. In a seminal paper [13], R. V. Moody and J. Patera pushed the theory of graded contractions of Lie algebras further with graded contractions of representations of Lie algebras. By considering along with graded Lie algebras their compatibly graded finite-dimensional representations, they obtained a theory of contractions of representations that contains the Lie algebra contractions as a special case for adjoint representation. This is unfortunately the only existing mathematical theory of this matter, and moreover it is not concerned with the question of which representations can be compatibly graded. Namely, compatibly graded finite-dimensional representations were assumed throughout the paper [13], Eqs. (2.10) and (2.11) which is a valid assumption if the grading is induced by an inner automorphism. In this respect they also provided a recipe for finding the corresponding grading of vector space $V$ on which the representation is acting (5). One should also mention a short note [15] on the subject, but up to now nobody has gone ahead with a further study of representations of Lie algebras related to their gradings, especially when the gradings are induced by outer automorphisms. We are aware that finite-dimensional representations of contracted Lie algebras can only be non-unitary. However, such representations, usually indecomposable, also have some interest in physics.

Our paper, as a starting point for such an investigation, gives answers under the restrictive assumptions used in [13]. Thus we restrict our consideration to

1. complex Lie algebras of type $A$,

2. finite-dimensional representations,

3. group gradings with the grading group $\mathbb{Z}_{2}$.

$\mathbb{Z}_{2}$-gradings are closely related to involutive (second order) automorphisms of Lie algebras. In physical applications they are especially useful as generalized parity transformations. In this connection our earlier paper [12] dealt with the well-known space-time parity transformations - space inversion and time reversal and the associated graded contractions for the de Sitter Lie algebras (type $B$ ). Here we start with the simplest case of finite-dimensional representations of classical Lie algebras of type $A$.

The paper is organized as follows. Section 2 is devoted to representations compatible with a grading. Explicit results are obtained in Section 3 for finite-dimensional representations of $\operatorname{sl}(n, \mathbb{C})$ compatible with $\mathbb{Z}_{2}$-gradings generated either by an inner automorphism of order 2 or by an outer automorphism of order 2 . Our concrete results are illustrated on the simple Lie algebra $\operatorname{sl}(3, \mathbb{C})$. 


\section{Representations compatible with grading}

\subsection{Graded contractions of Lie algebras}

A grading of a Lie algebra $L$ is a decomposition $\Gamma$ of the vector space $L$ into vector subspaces $L_{j}, j \in \mathcal{J}$, such that $L$ is a direct sum of these subspaces $L_{j}$, and, for any pair of indices $j, k \in \mathcal{J}$, there exists $l \in \mathcal{J}$ such that $\left[L_{j}, L_{k}\right] \subseteq L_{l}$. We denote the grading by

$$
\Gamma: L=\bigoplus_{j \in \mathcal{J}} L_{j}
$$

(let us note that in our definition of grading we do not exclude trivial subspaces $L_{i}=\{0\}$ ). It follows directly from the definition that for any grading $\Gamma: \bigoplus_{j \in \mathcal{J}} L_{j}$ and any automorphism $g \in \mathcal{A} u t L$ the decomposition $\Gamma^{\prime}: \bigoplus_{j \in \mathcal{J}} g\left(L_{j}\right)$ is also a grading. Gradings $\Gamma$ and $\Gamma^{\prime}$ are called equivalent.

Now we describe a specific type of grading, namely a group grading. A grading $\Gamma: L=\oplus_{j \in \mathcal{J}} L_{j}$ is called a group grading if the index set $\mathcal{J}$ can be embedded into a semigroup $G$ (whose binary operation is denoted by + ), and, for any pair of indices $j, k \in \mathcal{J}$, it holds that

$$
\left[L_{j}, L_{k}\right] \subseteq L_{j+k}
$$

Since even trivial subspaces are generally allowed in the decomposition of $L$, the semigroup $G$ may be used as the index set of the group grading. In this case we will speak about a $G$-grading $\Gamma$. We will focus in this paper on group gradings only and we assume in the sequel that the indices of the grading subspaces belong to a group $G$, i.e. $\Gamma$ is a $G$-grading of $L$.

A grading $\Gamma: L=\bigoplus_{i \in \mathcal{J}} L_{i}$ of a Lie algebra $L$ is a starting point for the study of graded contractions of the Lie algebra. This method for finding contractions of Lie algebras was introduced in [11, 13]. In this type of contraction, we define new Lie brackets by the prescription

$$
[x, y]_{n e w}:=\varepsilon_{j, k}[x, y] \text {, where } x \in L_{j}, y \in L_{k} .
$$

The complex or real parameters $\varepsilon_{j, k}$ for $j, k \in G$ must be determined in such a way that the vector space $L$ with the binary operation $[., .]_{\text {new }}$ again forms a Lie algebra. Antisymmetry of Lie brackets demands that $\varepsilon_{j, k}=\varepsilon_{k, j}$. If, moreover, the coefficients $\varepsilon_{j, k}$ fulfill the first basic set of contraction equations [14]:

$$
\varepsilon_{i, j} \varepsilon_{i+j, k}=\varepsilon_{j, k} \varepsilon_{j+k, i}=\varepsilon_{k, i} \varepsilon_{k+i, j} \quad \text { for all } i, j, k \in G,
$$

then the vector space $L$ with new brackets $[x, y]_{n e w}$ satisfies the Jacobi identities as well. This new Lie algebra will be denoted by $L^{\varepsilon}$. Note that the equations (3) involve only relevant parameters for which the corresponding commutators $\left[L_{j}, L_{k}\right]$ do not vanish.

Example $1 \mathbb{Z}_{2}$-grading. The most notorious case of group grading is $\mathbb{Z}_{2}$-grading. ${ }^{1}$ Here a Lie algebra $L$ over $\mathbb{C}$ is decomposed into two non-zero grading subspaces $L_{0}$ and $L_{1}$, where

$$
0 \neq\left[L_{0}, L_{0}\right] \subseteq L_{0}, \quad 0 \neq\left[L_{0}, L_{1}\right] \subseteq L_{1}, \quad 0 \neq\left[L_{1}, L_{1}\right] \subseteq L_{0} .
$$

Here we have applied the generic condition that in each class of commutators there exists at least one nonvanishing commutator. For a $\mathbb{Z}_{2}$-grading of a Lie algebra $L$, the generic system of equations (3) has a very simple form

$$
\left(\varepsilon_{00}-\varepsilon_{01}\right) \varepsilon_{01}=0=\left(\varepsilon_{00}-\varepsilon_{01}\right) \varepsilon_{11}, \quad \varepsilon_{10}=\varepsilon_{01} .
$$

There exist infinitely many solutions $\varepsilon=\left(\varepsilon_{j k}\right)$ of this system. However for many solutions, the contracted algebras $L^{\varepsilon}$ are isomorphic. It can be shown that only four solutions

$$
\left(\varepsilon_{j k}\right)=\left(\begin{array}{cc}
1 & 1 \\
1 & 0
\end{array}\right), \quad\left(\begin{array}{ll}
1 & 0 \\
0 & 0
\end{array}\right), \quad\left(\begin{array}{ll}
0 & 0 \\
0 & 1
\end{array}\right), \quad \text { and } \quad\left(\begin{array}{ll}
0 & 0 \\
0 & 0
\end{array}\right)
$$

give mutually non-isomorphic Lie algebras $L^{\varepsilon}$ over $\mathbb{C}$. (The original Lie algebra is obtained with all parameters $\varepsilon_{i j}=1$.) The contracted algebra obtained by the first solution is the semidirect sum of $L_{0}$ with a commutative algebra $L_{1}$ and corresponds to the Inönü-Wigner contraction. The second solution is the direct sum of $L_{0}$ and the commutative algebra $L_{1}$. The third solution corresponds to the central extension of $L_{1}$ (considered as a commutative algebra) by the commutative algebra $L_{0}$. The fourth solution is an Abelian Lie algebra.

\footnotetext{
${ }^{1}$ Note that special $\mathbb{Z}_{2}$-graded contractions are closely related to Inönü-Wigner contractions [12].
} 


\subsection{Representations of graded contractions}

Let us focus on the question of a representation of the contracted Lie algebra $L^{\varepsilon}$. We will reformulate the method proposed in [13], which enables us to find a representation of $L^{\varepsilon}$ by modifying a given representation of the original algebra $L$. It involves a simultaneous grading of the Lie algebra $L$ and the representation space $V$.

Definition 2.1 Let $r: L \mapsto$ End $V$ be a representation of Lie algebra $L$ and let $\Gamma: L=\bigoplus_{i \in G} L_{i}$ be its $G$-grading. We say that the representation $r$ is compatible with the G-grading, if there exists a decomposition of the vector space $V$ into a direct sum $V=\oplus_{i \in G} V_{i}$ such that

$$
r\left(X_{i}\right) V_{j} \subset V_{i+j} \quad \text { for each } i, j \in G \text { and any } X_{i} \in L_{i} .
$$

Remark 2.2 Let $r$ be a representation of $L$ compatible with the grading $L=\oplus_{i \in G} L_{i}$ and $h \in \mathcal{A} u t L$ be any automorphism of $L$. Then $r \circ h^{-1}$ is a representation of $L$ compatible with the equivalent grading $L=\bigoplus_{i \in G} h\left(L_{i}\right)$, since $\left(r \circ h^{-1}\right) h\left(X_{i}\right) V_{j}=r\left(X_{i}\right) V_{j} \subset V_{i+j}$.

Suppose we are given a representation $r$ of $L$ compatible with the $G$-grading. We are looking for a representation $r^{\varepsilon}$ of a contracted Lie algebra $L^{\varepsilon}$. According to [13] we define

$$
r^{\varepsilon}\left(X_{i}\right) v_{j}:=\psi_{i, j} r\left(X_{i}\right) v_{j} \quad\left(\text { for each } i, j \in G, \quad \text { any } \quad X_{i} \in L_{i} \text { and any } v_{j} \in V_{j}\right. \text { ), }
$$

where $\psi_{i, j}$ are unknown parameters. The requirement that $r^{\varepsilon}$ is a representation of $L^{\varepsilon}$ formally means

$$
r^{\varepsilon}\left(\left[X_{i}, X_{j}\right]_{n e w}\right) v_{k}=\left[r^{\varepsilon}\left(X_{i}\right), r^{\varepsilon}\left(X_{j}\right)\right] v_{k}=\left(r^{\varepsilon}\left(X_{i}\right) r^{\varepsilon}\left(X_{j}\right)-r^{\varepsilon}\left(X_{j}\right) r^{\varepsilon}\left(X_{i}\right)\right) v_{k}
$$

for any $X_{i} \in L_{i}, X_{j} \in L_{j}$, and $v_{k} \in V_{k}$. Using equations (2) and (6) and relation (5) we obtain

$$
\psi_{j, k} \psi_{i, j+k} r\left(X_{i}\right) r\left(X_{j}\right)-\psi_{i, k} \psi_{j, i+k} r\left(X_{j}\right) r\left(X_{i}\right)=\varepsilon_{i, j} \psi_{i+j, k} r\left(\left[X_{i}, X_{j}\right]\right)
$$

Since $r$ is a representation of $L$, we know that $r\left(X_{i}\right) r\left(X_{j}\right)-r\left(X_{j}\right) r\left(X_{i}\right)=r\left(\left[X_{i}, X_{j}\right]\right)$. Therefore, the choice of parameters $\psi_{i, j}$ satisfying the second basic set of contraction equations [14]

$$
\psi_{j, k} \psi_{i, j+k}=\psi_{i, k} \psi_{j, i+k}=\varepsilon_{i, j} \psi_{i+j, k}
$$

implies that $r^{\varepsilon}$ defined by (6) is a representation of the contracted Lie algebra $L^{\varepsilon}$. Solutions of (7) determine the contractions of the chosen representations. Let us stress that, if $r\left(\left[X_{i}, X_{j}\right]=0\right.$ for all $X_{i} \in L_{i}, X_{j} \in L_{j}$, conditions (7) are not necessary.

Comparing (7) and (3) we see that the system of quadratic equations for parameters $\psi_{i, j}$ has at least one solution, namely $\psi_{i, j}=\varepsilon_{i, j}$ for each pair $i, j$ (adjoint representation of $L^{\varepsilon}$ ). Therefore the mapping $r^{\varepsilon}: L^{\varepsilon} \mapsto$ End $V$ defined by (6) is a representation of the graded Lie algebra $L^{\varepsilon}$. Usually, there also exist other solutions of the system (7), and therefore more representations of the same contracted algebra $L^{\varepsilon}$.

Example $2 \mathbb{Z}_{2}$-graded representation. Consider a $\mathbb{Z}_{2}$-grading of a Lie algebra $L$ and its representation $r$ which is compatible with the grading. For the corresponding decomposition of the vector space $V=V_{0} \oplus V_{1}$ we may construct a basis $\mathcal{B}$ of $V$ composed of the basis of $V_{0}$ and the basis of $V_{1}$. In such a basis $\mathcal{B}$, the grading relations (4) acquire the block form explicitly

$$
r\left(X_{0}\right)=\left(\begin{array}{cc}
A\left(X_{0}\right) & 0 \\
0 & B\left(X_{0}\right)
\end{array}\right) \quad \text { and } \quad r\left(X_{1}\right)=\left(\begin{array}{cc}
0 & C\left(X_{1}\right) \\
D\left(X_{1}\right) & 0
\end{array}\right) .
$$

In the sequel, we will illustrate all notions on the Lie algebra $L^{\varepsilon}$ obtained by contraction from a $\mathbb{Z}_{2}$-grading of a Lie algebra $L$ by the first solution

$$
\left(\varepsilon_{j k}\right)=\left(\begin{array}{cc}
1 & 1 \\
1 & 0
\end{array}\right)
$$

given in Example 1. For this Lie algebra $L^{\varepsilon}$ the commutation relations have the form

$$
[x, y]_{\text {new }}=[x, y] \text {, if } x, y \in L_{0} \text { or if } x \in L_{0}, y \in L_{1} \quad \text { and } \quad[x, y]_{\text {new }}=0 \text {, if } x, y \in L_{1} \text {. }
$$

In this case the system of equations $(7)$ is

$$
\psi_{00} \psi_{00}=\psi_{00}, \quad \psi_{10} \psi_{01}=\psi_{00} \psi_{10}=\psi_{10}
$$




$$
\begin{gathered}
\psi_{01} \psi_{01}=\psi_{01}, \quad \psi_{11} \psi_{00}=\psi_{01} \psi_{11}=\psi_{11}, \\
\psi_{10} \psi_{11}=0
\end{gathered}
$$

All solutions (up to equivalence of representations) of this system are

$$
\left(\psi_{j k}\right)=\left(\begin{array}{cc}
1 & 1 \\
1 & 0
\end{array}\right),\left(\begin{array}{ll}
1 & 1 \\
0 & 1
\end{array}\right),\left(\begin{array}{ll}
1 & 1 \\
0 & 0
\end{array}\right),\left(\begin{array}{ll}
1 & 0 \\
0 & 0
\end{array}\right),\left(\begin{array}{ll}
0 & 1 \\
0 & 0
\end{array}\right) \text { and }\left(\begin{array}{ll}
0 & 0 \\
0 & 0
\end{array}\right)
$$

The representations $r^{\varepsilon}$ of the contracted Lie algebra $L^{\varepsilon}$ in the chosen basis $\mathcal{B}$ of the vector space $V$ have the block form

$$
r^{\varepsilon}\left(X_{0}\right)=\left(\begin{array}{cc}
\psi_{00} A\left(X_{0}\right) & 0 \\
0 & \psi_{01} B\left(X_{0}\right)
\end{array}\right) \quad \text { and } \quad r^{\varepsilon}\left(X_{1}\right)=\left(\begin{array}{cc}
0 & \psi_{11} C\left(X_{1}\right) \\
\psi_{10} D\left(X_{1}\right) & 0
\end{array}\right),
$$

where for parameters $\left(\psi_{i j}\right)$ one may choose one of the six solutions. Let us mention that only the first two solutions are interesting since the elements of subalgebra $L_{1}$ are represented by zero operators in the remaining solutions.

\subsection{Group gradings and automorphisms}

The simplest way to find a group grading of a Lie algebra is to decompose the vector space $L$ into eigensubspaces of a diagonalizable automorphism $g \in \mathcal{A} u t L[6]$. For any pair of its eigenvectors $x_{\lambda}$ and $x_{\mu}$ corresponding to eigenvalues $\lambda$ and $\mu$, respectively, we have

$$
g\left(\left[x_{\lambda}, x_{\mu}\right]\right)=\left[g\left(x_{\lambda}\right), g\left(x_{\mu}\right)\right]=\lambda \mu\left[x_{\lambda}, x_{\mu}\right] .
$$

Thus the commutator $\left[x_{\lambda}, x_{\mu}\right]$ is either zero or an eigenvector corresponding to the eigenvalue $\lambda \mu$. Let us denote by $\sigma(g)$ the spectrum of automorphism $g$ and by $L_{\lambda}$ the eigensubspace corresponding to $\lambda \in \sigma(g)$. The decomposition

$$
\Gamma: L=\bigoplus_{\lambda \in \sigma(g)} L_{\lambda}
$$

is a group grading, where the multiplicative semigroup generated by the spectrum of $g$ can be taken as a semigroup $G$.

Remark 2.3 If $h \in \mathcal{A}$ ut $L$, then the decomposition of $L$ into eigensubspaces of the automorphism $h g h^{-1}$ is $L=\bigoplus_{\lambda \in \sigma(g)} h\left(L_{\lambda}\right)$, i.e. the gradings given by conjugated automorphisms $g$ and $h g h^{-1}$ are equivalent. Therefore, the automorphisms $g$ and $h g h^{-1}$ are called equivalent as well. Note however that different inequivalent automorphisms may even give the same grading.

Similarly, if $g_{1}, g_{2}, \ldots, g_{r}$ are mutually commuting automorphisms of $L$, then the decomposition of $L$ into common eigensubspaces of all these automorphisms is a group grading of $L$. The semigroup suitable for indexing this grading is $G_{1} \times G_{2} \times \ldots \times G_{r}$, where each $G_{i}$ is the semigroup generated by the spectrum of the automorphism $g_{i}$.

Furthermore, for Lie algebras over the complex field $\mathbb{C}$, any group grading can be obtained by this procedure. Let us emphasize that this is not the case for real Lie algebras. In the following, in order to study the compatibility problem, we shall consider the simplest case of group grading determined by one automorphism.

\subsection{Group grading determined by one automorphism}

Let $\Gamma$ be a grading of the form (8), i.e. obtained by decomposition of $L$ into eigensubspaces of a single automorphism $g$. We may assume that $g$ has a finite order, say $g^{k}=I d$. For its spectrum we have

$$
\sigma(g) \subset\left\{e^{i \frac{2 \pi}{k} \ell} \mid \ell=0,1,2, \ldots, k-1\right\}=: G .
$$

This means that $\Gamma$ is a $G$-grading.

Let us consider an irreducible $d$-dimensional representation $r$ of the Lie algebra $L$. Our aim is to discuss the question of compatibility of $r$ with $G$-grading. Let $R_{g}$ be a non-singular matrix in $\mathbb{C}^{d \times d}$ such that

$$
r(g(x))=R_{g} r(x) R_{g}^{-1} \text { for all } x \in L .
$$


As $g^{k}=I d$, the previous equality gives

$$
r(x)=r\left(g^{k}(x)\right)=R_{g}^{k} r(x) R_{g}^{-k} \quad \text { or } \quad\left[R_{g}^{k}, r(x)\right]=0 \quad \text { for all } x \in L .
$$

Since the representation $r$ is irreducible, by Schur's lemma $R_{g}^{k}=\alpha I d$ for some $\alpha \in \mathbb{C}$. Of course, any nonzero multiple of $R_{g}$ also satisfies the relation (10). Therefore without loss of generality, we may assume that

$$
R_{g}^{k}=I d, \quad \text { where } k \text { is the order of automorphism } g .
$$

This normalization guarantees that the spectrum of matrix $R_{g}$ and the spectrum of automorphism $g$ belong to the same group $G$. In particular, since $R_{g}^{k}$ is the identity, matrix $R_{g}$ is diagonalizable. Let $V=\oplus_{\lambda \in G} V_{\lambda}$ denote the decomposition of column space $\mathbb{C}^{d}$ into eigensubspaces of matrix $R_{g}$, i.e. $R_{g} v_{\lambda}=\lambda v_{\lambda}$ for all $v_{\lambda} \in V_{\lambda}$. We will show that this decomposition is exactly the decomposition required in Definition 2.1.

Let us consider some $\mu \in \sigma(g)$ so that $g\left(x_{\mu}\right)=\mu x_{\mu}$ for all $x_{\mu} \in L_{\mu}$. Relation (10) for $x=x_{\mu}$ leads to the matrix relation

$$
r\left(g\left(x_{\mu}\right)\right) R_{g}=r\left(\mu x_{\mu}\right) R_{g}=\mu r\left(x_{\mu}\right) R_{g}=R_{g} r\left(x_{\mu}\right)
$$

which acts on a column vector $v_{\lambda} \in V_{\lambda}$ as

$$
\mu \lambda r\left(x_{\mu}\right) v_{\lambda}=R_{g} r\left(x_{\mu}\right) v_{\lambda}
$$

The last equality means that the column $r\left(x_{\mu}\right) v_{\lambda}$ is either zero or it is an eigenvector of matrix $R_{g}$ corresponding to eigenvalue $\mu \lambda$. Therefore

$$
r\left(x_{\mu}\right) V_{\lambda} \subset V_{\mu \lambda} \quad \text { for any } \lambda, \mu \in G \text { and any } x_{\mu} \in L_{\mu} .
$$

This is relation (5) written in the multiplicative form. Of course, our multiplicative group $G$ defined in (9) is isomorphic to the additive group $\mathbb{Z}_{k}$.

We have seen that matrix $R_{g}$ with the properties (10) and (11) guarantees the compatibility of grading of $L$ with the representation of the Lie algebra $L$. Such matrix $R_{g}$ will be called the simulation matrix of automorphism $g$. Matrix $R_{g}$ depends on the chosen automorphism $g$ and on the chosen representation $r$. The idea for finding the simulation matrix is more straightforward if $g \in \mathcal{A} u t L$ is an inner automorphism. In this case it is natural to search for $R_{g}$ among matrices in the representation of the corresponding Lie group. This idea was already presented in [11] and [15], where $R_{g}$ was a representation of an element of finite order [9]. Nevertheless, we show that it is also possible to find the simulation matrix $R_{g}$ even for an outer automorphism $g$. In the sequel, we will concentrate on the Lie algebras $\operatorname{sl}(n, \mathbb{C})$. The reason is that these algebras (with the exception of $o(8, \mathbb{C})$ ) are the only simple classical Lie algebras over $\mathbb{C}$ for which the group of automorphisms contains an outer automorphism as well [7].

\section{Representations of $s l(n, \mathbb{C})$ compatible with $\mathbb{Z}_{2}$-grading}

We will identify the Lie algebra $\operatorname{sl}(n, \mathbb{C})$ with $\left\{X \in \mathbb{C}^{n \times n} \mid \operatorname{tr} X=0\right\}$. Any $\mathbb{Z}_{2}$-grading of it is uniquely related to an automorphism of order 2. Let us therefore recall the structure of $\mathcal{A} u t \operatorname{sl}(n, \mathbb{C})$ as described in [7]:

1. for any inner automorphism $g$ there exists a matrix $A \in S L(n, \mathbb{C}):=\left\{A \in \mathbb{C}^{n \times n} \mid \operatorname{det} A=1\right\}$ such that

$$
g(X)=A d_{A} X=A X A^{-1} \quad \text { for any } X \in \operatorname{sl}(n, \mathbb{C}) ;
$$

2. the mapping given by the prescription

$$
\text { Out }_{I} X:=-X^{T} \text { for any } X \in \operatorname{sl}(n, \mathbb{C})
$$

is an outer automorphism of order 2;

3. any outer automorphism $g$ is a composition of an inner automorphism and the automorphism $\mathrm{Out}_{I}$.

4. to any outer automorphism $O u t_{A}$ of order two there exists an inner automorphism $A d_{P}$ such that $A d_{P}^{-1}$ Out $_{A} A d_{P}=$ Out $_{P^{T} A P}=$ Out $_{I}$, i.e. Out $A$ and Out $_{I}$ are equivalent (see [6], Lemma A.1). 
The next ingredient for the construction of simulation matrices of automorphisms is the knowledge of finitedimensional irreducible representations of $\operatorname{sl}(n, \mathbb{C})$. These representations are well described by Gel'fand-Tseitlin formalism $[4,10,2]$. Any irreducible representation $r$ of $\operatorname{sl}(n, \mathbb{C})$ is in one-to-one correspondence with an $n$-tuple $\left(m_{1, n}, m_{2, n}, \ldots, m_{n, n}\right)$ of non-negative integer parameters $m_{1, n} \geq m_{2, n} \geq \ldots \geq m_{n, n}=0$. The dimension of the representation space of $r=r\left(m_{1, n}, m_{2, n}, \ldots, m_{n, n}\right)$ is given by the number of triangular patterns

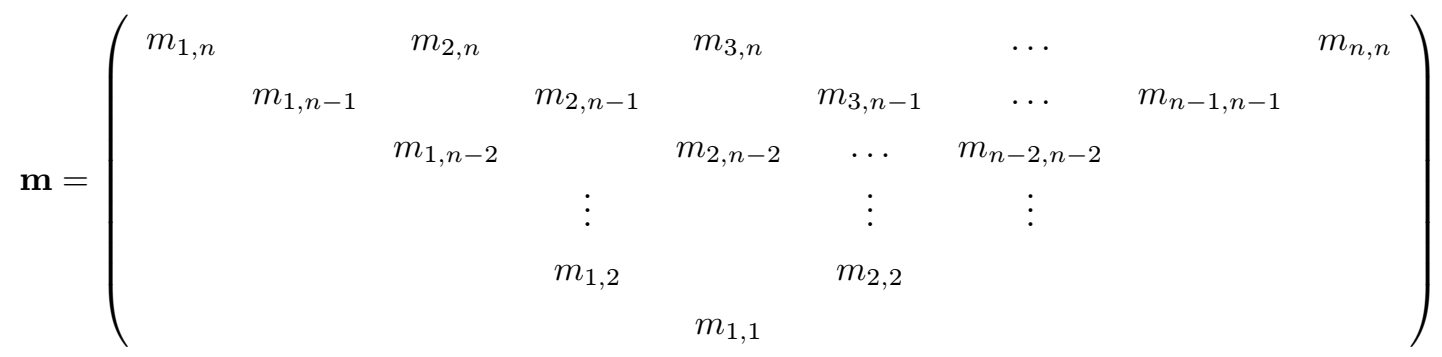

in which the numbers $m_{i, j} \in \mathbb{Z}$ satisfy $m_{i, j+1} \geq m_{i, j} \geq m_{i+1, j+1}$ for all $1 \leq i \leq j \leq n-1$. To any such pattern $\mathbf{m}$, we assign the basis vector $\xi(\mathbf{m})$. The representation $r$ is fully determined by the action $r\left(E_{k \ell}\right)$ on all basis vectors $\xi(\mathbf{m})$ for any $k, \ell=1,2, \ldots, n$. (We have adopted the notation $E_{k \ell}$ for $n \times n$ matrices with elements $\left(E_{k \ell}\right)_{i j}=\delta_{i k} \delta_{\ell j}$. $)$ This action can be found e.g. in [4], but for the reader's convenience the representation of $g l(n, \mathbb{C})$ is described in the Appendix.

\subsection{Inner automorphisms of order two}

Any inner automorphism $g$ of order two is associated by the equality $g=A d_{A}$ with a group element $A \in S L(n, \mathbb{C})$ such that $A$ does not belong to the center $\mathcal{Z}[S L(n, \mathbb{C})]$ and $A^{2}$ belongs to the center. If we denote $\omega=e^{\frac{i \pi}{n}}$, then the center can be written explicitly $\mathcal{Z}[S L(n, \mathbb{C})]=\left\{\omega^{2 \ell} I_{n} \mid \ell=0,1, \ldots, n-1\right\}$. A simple calculation shows that any such element $A \in S L(n, \mathbb{C})$ is up to equivalence one of the matrices

$$
A_{n, s}:=\omega^{\eta(s)}\left(\begin{array}{cc}
I_{n-s} & 0 \\
0 & -I_{s}
\end{array}\right) \text { where } s=1, \ldots,\left\lfloor\frac{n}{2}\right\rfloor \text { and } \eta(s)=\left\{\begin{array}{cl}
0 & \text { if } s \text { is even } \\
1 & \text { if } s \text { is odd }
\end{array}\right.
$$

(Note that $A_{n, 0}=I_{n}$ belongs to $\mathcal{Z}[S L(n, \mathbb{C})]$.) These matrices may be rewritten by using elements of the Lie algebra $\operatorname{sl}(n, \mathbb{C})$ as follows:

$$
A_{n, s}=\exp \left(X_{n, s}\right) \quad \text { with } X_{n, s}=i \pi\left(\begin{array}{cc}
\frac{\eta(s)}{n} I_{n-s} & 0 \\
0 & \frac{\eta(s)}{n} I_{s}+M_{s}
\end{array}\right),
$$

where $M_{s}=\operatorname{diag}\left(-1,1,-1, \ldots,(-1)^{s}\right) \in \mathbb{C}^{s \times s}$. One can use the notation of $E_{k k}$ and write

$$
X_{n, s}=i \pi\left(\frac{\eta(s)}{n} \sum_{k=1}^{n} E_{k k}+\sum_{k=n-s+1}^{n}(-1)^{n-s+1-k} E_{k k}\right) .
$$

If $r$ is any representation of the Lie algebra $\operatorname{sl}(n, \mathbb{C})$, then $R_{A_{n, s}}:=\exp \left(r\left(X_{n, s}\right)\right)$ satisfies

$$
R_{A_{n, s}} r(X)\left(R_{A_{n, s}}\right)^{-1}=r\left(A_{n, s} X A_{n, s}^{-1}\right)=r\left(A d_{A_{n, s}} X\right) \quad \text { and } \quad\left(R_{A_{n, s}}\right)^{2}=I d .
$$

Therefore, matrix $R_{A_{n, s}}$ is the simulation matrix of the inner automorphism $g=A d_{A_{n, s}}$. We have shown

Theorem 3.1 Any $\mathbb{Z}_{2}$-grading of the Lie algebra $\operatorname{sl}(n, \mathbb{C})$ obtained by an inner automorphism and any irreducible representation of $\operatorname{sl}(n, \mathbb{C})$ are compatible.

Using (12) and the explicit form of the Gel'fand-Tseitlin representation we obtain for any basis vector $\xi(\mathbf{m})$

$$
r\left(X_{n, s}\right) \xi(\mathbf{m})=i \pi\left(\frac{\eta(s)}{n} r_{n}(\mathbf{m})+2 \sum_{k=1}^{s-1}(-1)^{k-1} r_{n-s+k}(\mathbf{m})-r_{n-s}(\mathbf{m})-(-1)^{\eta(s)} r_{n}(\mathbf{m})\right) \xi(\mathbf{m}) .
$$

Thus we have arrived at the explicit form of the simulation matrix of the automorphism $g=A d_{A_{n, s}}$

$$
R_{A_{n, s}} \xi(\mathbf{m})=e^{i \pi\left(\left(\frac{\eta(s)}{n}-1\right) r_{n}(\mathbf{m})-r_{n-s}(\mathbf{m})\right)} \xi(\mathbf{m})
$$




\subsection{Outer automorphism of order two}

As explained at the beginning of Section 3 , any outer automorphism of order two on $\operatorname{sl}(n, \mathbb{C})$ is up to equivalence the automorphism $\operatorname{Out}_{I}(X)=-X^{T}$, and thus we will focus only on it without loss of generality.

It is well known that for an irreducible representation $r$ characterized in the Gel'fand-Tseitlin formalism by the $n$-tuple $\left(m_{1, n}, m_{2, n}, \ldots, m_{n, n}\right)$, the mapping $-r^{T}$ (to minus transposed matrices) is also an irreducible representation. This representation is equivalent to the contragredient representation $r^{c}$, which is characterized by the $n$-tuple $\left(m_{1, n}^{\prime}, m_{2, n}^{\prime}, \ldots, m_{n, n}^{\prime}\right)$, where

$$
m_{i, n}^{\prime}=m_{1, n}-m_{n-i+1, n} \quad \text { for } i=1,2, \ldots, n .
$$

Let us consider a triangular pattern $\mathbf{m}$ filled by indices $m_{i, j}, 1 \leq i \leq j \leq n$, and associated with the basis vector $\xi(\mathbf{m})$ of representation $r$. To any such pattern $\mathbf{m}$, we may assign the unique triangular pattern $\mathbf{m}^{\prime}$ with indices $m_{i, j}^{\prime}:=m_{1, n}-m_{j-i+1, j}$. It is easy to check that $m_{i, j}^{\prime}$ satisfies the necessary inequalities for $\mathbf{m}^{\prime}$ to be a correct pattern of the contragredient representation $r^{c}$. Let us define the linear mapping $J$ of the representation space of $r$ onto the representation space of $r^{c}$ by

$$
J \xi(\mathbf{m}):=(-1)^{\sum_{i, j} m_{i, j}} \xi\left(\mathbf{m}^{\prime}\right) .
$$

On the other hand, from the formulae in the Appendix one sees that

$$
r^{T}\left(E_{i j}\right)=r\left(E_{j i}\right)=r\left(E_{i j}^{T}\right) .
$$

Using this fact one can prove by direct verification that the mapping $J$ satisfies

$$
-J r^{T}(X)=r^{c}(X) J \text { for any } X \in \operatorname{sl}(n, \mathbb{C}) .
$$

Let us return to our original task. We are looking for the simulation matrix of the automorphism $g=O u t_{I}$, i.e., we are looking for a matrix $R_{g}$ of order two such that

$$
r\left(O u t_{I}(X)\right)=-r\left(X^{T}\right)=R_{g} r(X) R_{g}^{-1} .
$$

According to (13), we have $r\left(X^{T}\right)=r^{T}(X)$ and therefore the existence of the simulation matrix $R_{g}$ means equivalence of the representations $r$ and $-r^{T}$, i.e. equivalence of $r$ and its contragredient representation $r_{c}$. The Gel'fand-Tseitlin result states that this is possible if and only if $n$-tuples $\left(m_{1, n}, m_{2, n}, \ldots, m_{n, n}\right)$ and $\left(m_{1, n}^{\prime}, m_{2, n}^{\prime}, \ldots, m_{n, n}^{\prime}\right)$ coincide. In this case the simulation matrix $R_{g}$ is equal to $J$. We have deduced

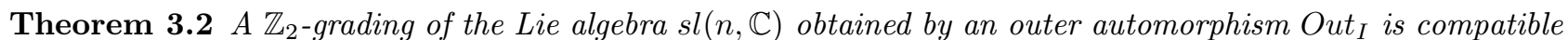
with an irreducible representation $r$ of $\operatorname{sl}(n, \mathbb{C})$ if and only if the representation is self-contragredient.

If we do not insist on the irreducibility of representation $r$, the class of representations compatible with the $\mathbb{Z}_{2}$ grading obtained by the automorphism $O u t_{I}$ is larger. Of course, if for a representation $r_{1}$ it is possible to find a simulation matrix $R^{(1)}$ and for a representation $r_{2}$ a simulation matrix $R^{(2)}$, then the direct sum $R^{(1)} \oplus R^{(2)}$ is the simulation matrix for the direct sum $r_{1} \oplus r_{2}$. To avoid a discussion of all such obvious cases, we will describe only those representations $r$ with simulation matrices $R$ for which the operator set $\{R\} \cup\{r(X) \mid X \in \operatorname{sl}(n, \mathbb{C})\}$ is irreducible, whereas the set $\{r(X) \mid X \in \operatorname{sl}(n, \mathbb{C})\}$ is reducible.

If $r_{0}$ is a $d$-dimensional irreducible representation of $\operatorname{sl}(n, \mathbb{C})$ then the $2 d$-dimensional representation $r:=$ $r_{0} \oplus\left(-r_{0}^{T}\right)$ assigns to $X$ the matrix

$$
r(X)=\left(\begin{array}{cc}
r_{0}(X) & 0 \\
0 & -\left(r_{0}(X)\right)^{T}
\end{array}\right)
$$

and therefore

$$
r\left(\text { Out }_{I}(X)\right)=\left(\begin{array}{cc}
-\left(r_{0}(X)\right)^{T} & 0 \\
0 & r_{0}(X)
\end{array}\right)=\left(\begin{array}{cc}
0 & I_{d} \\
I_{d} & 0
\end{array}\right) r(X)\left(\begin{array}{cc}
0 & I_{d} \\
I_{d} & 0
\end{array}\right)
$$

The matrix $\left(\begin{array}{cc}0 & I_{d} \\ I_{d} & 0\end{array}\right)$ is the simulation matrix of $O u t_{I}$. It is easy to see that the simulation matrix together with all $r(X)$ form an irreducible set. 


\section{3 $\mathbb{Z}_{2}$-grading of $\operatorname{sl}(3, \mathbb{C})$}

Let us illustrate the conclusions of the previous sections on the Lie algebra $\operatorname{sl}(3, \mathbb{C})$. On this algebra there exist only two inequivalent automorphisms of order two. In our notation $g_{1}=A d_{A_{3,1}}$ with

$$
A_{3,1}=\omega\left(\begin{array}{ccc}
1 & 0 & 0 \\
0 & 1 & 0 \\
0 & 0 & -1
\end{array}\right), \text { where } \omega=e^{\frac{i \pi}{3}}
$$

and $g_{2}=O u t_{I}$. The corresponding $\mathbb{Z}_{2}$-gradings are

$$
\begin{gathered}
\Gamma_{1}: \operatorname{sl}(3, \mathbb{C})=\left\{\left(\begin{array}{rrr}
a & b & 0 \\
c & d & 0 \\
0 & 0 & -a-d
\end{array}\right) \mid a, b, c, d \in \mathbb{C}\right\} \oplus\left\{\left(\begin{array}{lll}
0 & 0 & a \\
0 & 0 & b \\
c & d & 0
\end{array}\right) \mid a, b, c, d \in \mathbb{C}\right\}, \\
\Gamma_{2}: \operatorname{sl}(3, \mathbb{C})=\left\{\left(\begin{array}{rrr}
0 & a & b \\
-a & 0 & c \\
-b & -c & 0
\end{array}\right) \mid a, b, c \in \mathbb{C}\right\} \oplus\left\{\left(\begin{array}{ccc}
a & b & c \\
b & d & e \\
c & e & -a-d
\end{array}\right) \mid a, b, c, d, e \in \mathbb{C}\right\} .
\end{gathered}
$$

The first grading $\Gamma_{1}$ is compatible with any irreducible representation. The simulation matrix $R_{g_{1}}$ of the automorphism $g_{1}=A d_{A_{31}}$ acts on the Gel'fand-Tseitlin triangular patterns as follows

$$
R_{g_{1}}\left(\begin{array}{ccc}
m_{1,3} & m_{2,3} & 0 \\
m_{1,2} & m_{2,2} \\
m_{1,1}
\end{array}\right)=e^{-\frac{2 i \pi}{3}\left(m_{1,3}+m_{2,3}\right)} e^{-i \pi\left(m_{1,2}+m_{2,2}\right)}\left(\begin{array}{ccc}
m_{1,3} & m_{2,3} & 0 \\
m_{1,2} & m_{2,2} \\
m_{1,1} &
\end{array}\right) .
$$

The irreducible representations compatible with the second grading are only self-contragredient representations, i.e., representations $r=r(2 \ell, \ell, 0)$. In such representation, the operator $J$ is defined by

$$
J\left(\begin{array}{ccc}
2 \ell & \ell & 0 \\
m_{1,2} m_{2,2} \\
m_{1,1}
\end{array}\right)=(-1)^{\ell+m_{1,2}+m_{2,2}+m_{1,1}}\left(\begin{array}{ccc}
2 \ell & \ell & 0 \\
2 \ell-m_{2,2} & 2 \ell-m_{1,2} \\
2 \ell-m_{1,1}
\end{array}\right) .
$$

The lowest-dimensional non-trivial self-contragredient representation is $r=r(2,1,0)$, in fact, the adjoint representation. Its dimension is 8 and has the following explicit form on the basis vectors:

$$
\begin{aligned}
& R_{g_{2}}\left(\begin{array}{ccc}
2 & 1 & 0 \\
2 & 1 \\
2
\end{array}\right)=\left(\begin{array}{ccc}
2 & 1 & 0 \\
1 & 0 \\
0
\end{array}\right), \quad R_{g_{2}}\left(\begin{array}{ccc}
2 & 1 & 0 \\
1 & 0 \\
0
\end{array}\right)=\left(\begin{array}{ccc}
2 & 1 & 0 \\
2 & 1 \\
2
\end{array}\right), \quad R_{g_{2}}\left(\begin{array}{ccc}
2 & 1 & 0 \\
2 & 1 \\
1
\end{array}\right)=-\left(\begin{array}{ccc}
2 & 1 & 0 \\
1 & 0 \\
1
\end{array}\right), \\
& R_{g_{2}}\left(\begin{array}{ccc}
2 & 1 & 0 \\
1 & 0 \\
1
\end{array}\right)=-\left(\begin{array}{ccc}
2 & 1 & 0 \\
2 & 1 \\
1
\end{array}\right), \quad R_{g_{2}}\left(\begin{array}{ccc}
2 & 1 & 0 \\
2 & 0 \\
2
\end{array}\right)=-\left(\begin{array}{ccc}
2 & 1 & 0 \\
2 & 0 \\
0
\end{array}\right), \quad R_{g_{2}}\left(\begin{array}{ccc}
2 & 1 & 0 \\
2 & 0 \\
0
\end{array}\right)=-\left(\begin{array}{ccc}
2 & 1 & 0 \\
2 & 0 \\
2
\end{array}\right), \\
& R_{g_{2}}\left(\begin{array}{ccc}
2 & 1 & 0 \\
1 & 1 \\
1
\end{array}\right)=\left(\begin{array}{ccc}
2 & 1 & 0 \\
1 & 1 \\
1
\end{array}\right), \quad R_{g_{2}}\left(\begin{array}{ccc}
2 & 1 & 0 \\
2 & 0 \\
1
\end{array}\right)=\left(\begin{array}{ccc}
2 & 1 & 0 \\
2 & 0 \\
1 &
\end{array}\right) \text {. }
\end{aligned}
$$

If the representation $r=r\left(m_{13}, m_{23}, 0\right)$ is not self-contragredient, then grading $\Gamma_{2}$ is compatible with the reducible representation

$$
r_{\oplus}(X):=\left(\begin{array}{cc}
r(X) & 0 \\
0 & -(r(X))^{T}
\end{array}\right)
$$

and the corresponding simulation matrix on the double-dimensional space is $J=\sigma_{1} \otimes I$, where $I$ is the identity operator on the representation space of representation $r$ and $\sigma_{1}$ denotes the first Pauli matrix. 


\section{Conclusions}

Since basic concepts connected with gradings of Lie algebras were laid down already in the work of J. Patera and H. Zassenhaus [16], including the notion of compatibly graded representation, it is really surprising that there does not yet exist a theory of representations of graded Lie algebras compatible with a given grading. This work is devoted to first steps in investigating which irreducible representations of a Lie algebra $L$ are compatible with its $G$-grading, at least in a rather restricted framework. The main contribution of the paper consists in elucidating which representations of classical Lie algebras of type $A$ are compatible with a $\mathbb{Z}_{2}$-grading. Concretely, the results are as follows: if the involutive automorphism producing the $\mathbb{Z}_{2}$-grading is inner, then every irreducible finite-dimensional representation is compatible with the grading, but if the automorphism producing the grading is not inner, then the only irreducible finite-dimensional representations compatible with the grading are the self-contragredient ones. For the outer automorphism there is also a possibility of reducible representations involving pairs of mutually contragredient irreducible representations. Thus it is not generally true that every irreducible representation can be compatibly graded. The $s l(3, \mathbb{C})$-case is enclosed to illustrate the process.

One of our future goals is to enlarge the family of gradings of $L$ for which one can decide about compatibility with representations of $L$. Another goal is to study representations of physical interest of the so-called kinematical groups of space-times. The possible Lie algebras $L$ of these groups were classified in [1]. A rather remarkable fact was found there that very simple conditions imposed by space inversion and time reversal on the generators very severely constrain the possible Lie algebras. This result was confirmed in [12] from the corresponding $\mathbb{Z}_{2} \times \mathbb{Z}_{2}$-contractions of the de Sitter Lie algebras. From this point of view it would be useful to identify the gradings implicitly present for instance in $[3,18]$ and in other papers where contractions of representations are studied.

\section{Acknowledgement}

The authors would like to express their gratitude to the referees for their careful reading of the manuscript and for their valuable critical comments, which have helped to improve the presentation. We are grateful to Vyacheslav Futorny for fruitful discussions on relations between the notions of grading and group grading, and to Jiř́ Patera for introducing us to the problems connected with representations of contracted Lie algebras. We acknowledge financial support from the grants MSM6840770039 and LC06002 of the Ministry of Education, Youth, and Sports of the Czech Republic.

\section{Appendix. The Gel'fand-Tseitlin formalism}

Let us give an explicit description of the irreducible representations of $g l(n, \mathbb{C})$ in the Gel'fand-Tseitlin formalism $[4,10,2]$. Any irreducible representation $r$ of $g l(n, \mathbb{C})$ is in one-to-one correspondence with an $n$-tuple $\left(m_{1, n}, m_{2, n}, \ldots, m_{n, n}\right)$ of non-negative integer parameters $m_{1, n} \geq m_{2, n} \geq \ldots \geq m_{n, n} \geq 0$. Since any $E_{k \ell}$ can be obtained by commutation relations from $E_{k, k}, E_{k, k-1}$ and $E_{k-1, k}$, only formulas for $r\left(E_{k, k}\right), r\left(E_{k, k-1}\right)$ and $r\left(E_{k-1, k}\right)$ are needed:

$$
r\left(E_{k, k}\right) \xi(\mathbf{m})=\left(r_{k}-r_{k-1}\right) \xi(\mathbf{m}),
$$

where $r_{k}=m_{1, k}+\ldots+m_{k, k}$ for $k=1,2, \ldots, n$ and $r_{0}=0$,

$$
r\left(E_{k, k-1}\right) \xi(\mathbf{m})=a_{k-1}^{1} \xi\left(\mathbf{m}_{k-1}^{1}\right)+\ldots+a_{k-1}^{k-1} \xi\left(\mathbf{m}_{k-1}^{k-1}\right),
$$

where $\mathbf{m}_{k-1}^{j}$ denotes the triangular pattern obtained from $\mathbf{m}$ replacing $m_{j, k-1}$ by $m_{j, k-1}-1$,

$$
a_{k-1}^{j}=\left[-\frac{\prod_{i=1}^{k}\left(m_{i, k}-m_{j, k-1}-i+j+1\right) \prod_{i=1}^{k-2}\left(m_{i, k-2}-m_{j, k-1}-i+j\right)}{\prod_{i \neq j}\left(m_{i, k-1}-m_{j, k-1}-i+j+1\right)\left(m_{i, k-1}-m_{j, k-1}-i+j\right)}\right]^{1 / 2}
$$

and

$$
r\left(E_{k-1, k}\right) \xi(\mathbf{m})=b_{k-1}^{1} \xi\left(\mathbf{m}_{k-1}^{1}\right)+\ldots+b_{k-1}^{k-1} \xi\left(\mathbf{m}_{k-1}^{k-1}\right),
$$

where $\mathbf{m}_{k-1}^{j}$ denotes the triangular pattern obtained from $\mathbf{m}$ replacing $m_{j, k-1}$ by $m_{j, k-1}+1$, and

$$
b_{k-1}^{j}=\left[-\frac{\prod_{i=1}^{k}\left(m_{i k}-m_{j, k-1}-i+j\right) \prod_{i=1}^{k-2}\left(m_{i, k-2}-m_{j, k-1}-i+j-1\right)}{\prod_{i \neq j}\left(m_{i, k-1}-m_{j, k-1}-i+j\right)\left(m_{i, k-1}-m_{j, k-1}-i+j-1\right)}\right]^{1 / 2} .
$$




\section{References}

[1] Bacry, H., Lévy-Leblond, J.-M.: Possible kinematics, J. Math. Phys. 9 (1968), 1605-1614.

[2] Barut, A. O., Raczka, R.: Theory of Group Representations and Applications, World Scientific, Singapore, 2000, Chap. 10.

[3] De Bièvre, S., Cishahayo, C.: On the contraction of the discrete series of SU(1,1), Ann. Inst. Fourier, 43 (1993), 551-567.

[4] Gel'fand, I. M., Tseitlin, M. L.: Finite-dimensional representations of the group of unimodular matrices, Dokl. Akad. Nauk SSSR 71 (1950), 825-828.

[5] Gilmore, R.: Lie Groups, Lie Algebras, and Some of Their Applications, Wiley, New York 1974, Chap. 10.

[6] Havlíček, M., Patera, J., Pelantová, E.: On Lie gradings II, Lin. Alg. Appl. 277 (1998), 97-125.

[7] Helgason, S.: Differential Geometry, Lie Groups, and Symmetric Spaces. Academic Press, New York 1978.

[8] Inönü, E., Wigner, E. P.: On the contraction of groups and their representations, Proc. Nat. Acad. Sci. U.S.A. 39 (1952), 510-525.

[9] Kac, V. G.: Automorphisms of finite order of semisimple Lie algebras, Funct. Anal. Appl. 3 (1969), 252-254.

[10] Lemire, F., Patera, J.: Formal analytic continuation of Gel'fand's finite dimensional representations of gl(n,C), J. Math. Phys. 20 (1979), 820-829.

[11] de Montigny, M., Patera, J.: Discrete and continuous graded contractions of Lie algebras and superalgebras, J. Phys. A: Math. Gen. 24 (1991), 525-549.

[12] de Montigny, M., Patera, J., Tolar, J.: Graded contractions and kinematical groups of space-time, J. Math. Phys. 35 (1994), 405-425.

[13] Moody, R. V., Patera, J.: Discrete and continuous graded contractions of representations of Lie algebras, J. Phys. A: Math. Gen. 24 (1991), 2227-2 258.

[14] Patera, J.: Graded contractions of Lie algebras, representations and tensor products, AIP Conference Proceedings, Vol. 266 (1992), 46-54.

[15] Patera, J., Tolar, J.: On gradings of Lie algebras and their representations, In Lie Theory and Its Applications in Physics II, (eds. H.-D. Doebner, V. K. Dobrev, J. Hilgert), World Scientific, Singapore 1998, $109-118$.

[16] Patera, J., Zassenhaus, H.: On Lie gradings I, Lin. Alg. Appl. 112 (1989), 87-159.

[17] Patera, J., Zassenhaus, H.: The Pauli matrices in $n$ dimensions and finest gradings of simple Lie algebras of type $A_{n-1}$, J. Math. Phys. 29 (1988), 665-673.

[18] Ström, S.: Construction of representations of the inhomogeneous Lorentz group by means of contraction of representations of the (1+4) de Sitter group, Arkiv för Fysik 30 (1965), 455-472.

Prof. Ing. Miloslav Havlíček, DrSc.

E-mail: miloslav.havlicek@fjfi.cvut.cz

Prof. Ing. Edita Pelantová, CSc.

E-mail: edita.pelantova@fjfi.cvut.cz

Prof. Ing. Jiří Tolar, DrSc.

E-mail: jiri.tolar@fjfi.cvut.cz

Doppler Institute, Faculty of Nuclear Sciences and Physical Engineering

Czech Technical University in Prague, Czech Republic 